\title{
RESEARCH/RESEARCHERS
}

\section{Silica-Coated SWNTs Form Unique Nanostructures}

Although carbon nanotubes (CNTs) have many properties, such as high thermal and electrical conductivities and mechanical strength, that are advantageous to nanotechnology applications, their strong affinity for one another limits their compatibility with other materials. Dispersal of CNTs in a nanocomposite therefore presents a serious processing challenge. A.R. Barron's research group at Rice University has previously coated single-walled CNTs (SWNTs) with silica, thereby eliminating the undesirable intermolecular attractive forces. However, this method requires the use of cationic surfactants, which are less common than anionic surfactants, because an acidic medium is used. Furthermore, this acidic method produces hydrofluoric acid (HF), which converts some of the silica coating to fluorosilicates. More recently, Barron and R. Colorado, both from the Department of Chemistry and Center for Nanoscale Science and Technology at Rice, have developed a method of coating

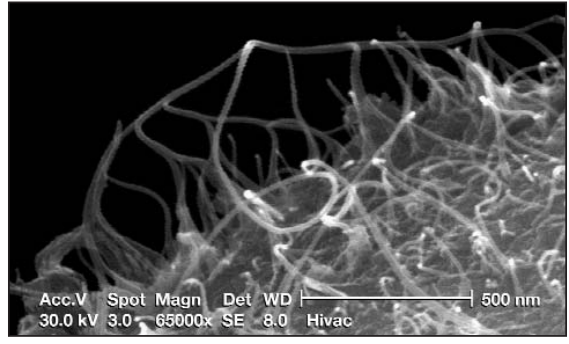

Figure 1. Scanning electron micrograph showing a complex structure consisting of welded $\mathrm{SiO}_{2}$-single-walled carbon nanotubes. Reprinted with permission from Chem. Mater. 16 (14) (July 13, 2004) p. 2692. (02004 American Chemical Society.

SWNTs with silica under basic conditions that are free of HF and can make use of the more common anionic surfactants.

As reported in the July 13 issue of Chemistry of Materials (p. 2691), the researchers adapted a previously published method for forming silica spheres and suspended SWNTs with a basic sodium sili- cate solution. Addition of ethanol induces the formation of silica acid, $\mathrm{Si}(\mathrm{OH})_{4}$, which quickly condenses onto SWNT surfaces to form polysilicic acid, that is, amorphous silica. The suspension consists of individual and bundled SWNTs, so the coated structures consist of individual and bundled SWNTs as well. Colorado and Barron also prepared films that they said formed mats composed of overlapping and interwoven coated tubes and bundles. The researchers said that scanning electron microscopy revealed a variety of unique nanostructures, including loops, curls, interconnects, and bridging structures (see Figure 1). Furthermore, the researchers showed that the coating process effectively fastens short tubes together to form longer structures. Colorado and Barron said that they are now "examining the potential of solutionbased methods of producing silica-coated SWNTs and mats for manipulating conducting/semiconducting SWNTs and insulating coatings to assemble useful nanoelectronic devices."

STEVEN TROHALAK

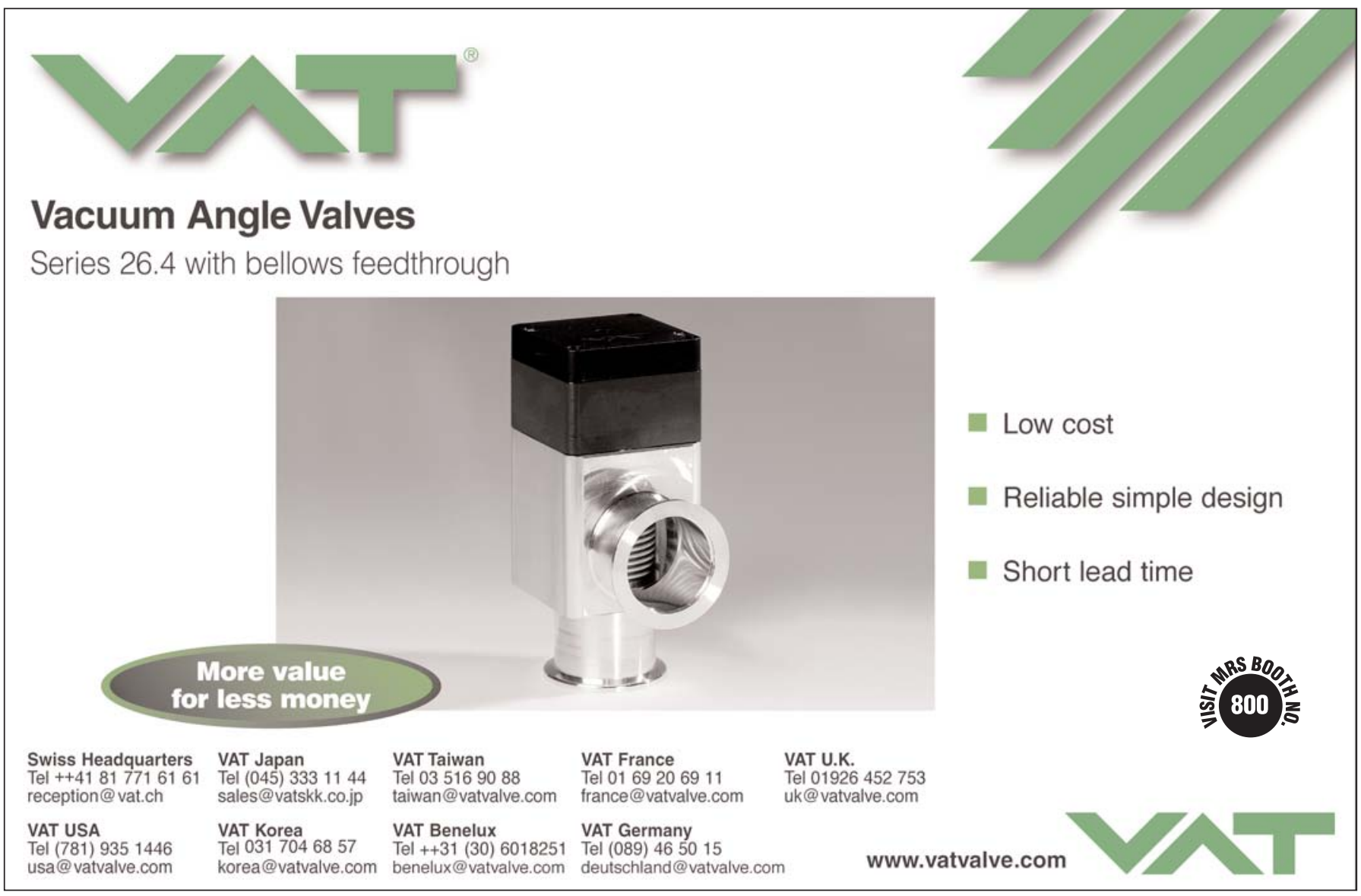

For more information, see http://advertisers.mrs.org 